\title{
Matgorzata Mazurek-Chwiejczak*
}

\section{TRENDS IN PERSONAL INCOME TAX PROGRESSION IN OECD COUNTRIES IN THE CONTEXT OF INCOME REDISTRIBUTION ${ }^{1}$}

\begin{abstract}
In recent years there has been an evident, widespread increase in income disparities in OECD countries. Progressive Personal Income Tax, which enables adjustment of the tax burden to individual's capacity to pay, is one of the fundamental instruments used in redistribution policy. The aim of the paper is comparative analysis of the level of Personal Income Tax (PIT) progression in OECD countries and identification of trends in progression in the context of income redistribution. The article discusses the progressivity level of PIT in OECD countries measured by the differences in the burden at different levels of income. The cross-country and historical trends in the statutory PIT rates, the number of tax brackets and the provisions which exempt an initial level of income from tax burden are analysed and graphically illustrated.
\end{abstract}

Keywords: Personal Income Tax, progression, redistribution, tax policy.

\section{INTRODUCTION}

Nowadays the gap between the richest and the poorest is at its highest level in most OECD countries. In 2014 the top $10 \%$ of population earned 9.5 times more than the bottom income decile. 30 years ago the ratio stood at 7:1 (OECD 2014a: 1). Over the last two or three decades the increase in top income shares has been observed in all but a few OECD countries (Matthews 2011: 18). Meanwhile the number of people at risk of poverty grew from $28 \%$ in 1990 to $34 \%$ in 2012. In Ireland, Spain and Portugal the ratio exceeds $40 \%$. The Gini coefficient on disposable income (after taxes and transfers) has increased since the mid-1980s by 3 percentage points to 0.32 (2011/2012).

The phenomenon has raised concerns about the impact of income and social inequalities on economies and societies. According to J.K. Galbraith (1996: 62-63) „the modern market economy accords wealth and distributes income in a highly

\footnotetext{
* Maria Curie-Skłodowska University (UMCS) in Lublin, malgorzata.mazurek-chwiejczak@ poczta.umcs.lublin.pl

${ }^{1}$ The project was funded by the National Science Centre based on the basis of the decision number DEC-2012/05/N/HS4/00481.
} 
unequal, socially adverse and functionally damaging fashion. (...) This, the good society cannot accept. (...) There is a strong chance that the more unequal the distribution of income, the more dysfunctional it becomes". The experiences of many countries prove that an excessive spread between the extremities of wealth and poverty gives rise to social conflicts and results in political upheavals (Moździerz 2012: 532). Moreover, there is strong evidence that the high level of income inequality affects economic growth. The latest OECD analysis suggests that the rise in Gini by 3 points would reduce GDP by 0.35 percentage points per year over 25 years. The cumulative negative impact seems to be significant -8.5 per cent GDP loss at the end of the period (OECD 2014a: 2).

That is why it is so important to have a properly defined policy for the narrowing of inequalities with a major part to be played by social transfers, as well as the tax system and its individual elements. The influence of fiscal policy on the level of income dispersion in OECD counties is significant. The comparison of the average value of Gini coefficient for the gross market income (i.e. before taxes and transfers) with its level for disposable income shows that the second indicator is definitely lower. The difference between these two coefficients in most OECD countries exceeds 0.15 (OECD 2014b). This proves the existence of widely defined fiscal policy and its influence on the level of income disparities.

Fiscal instruments of reduction of income inequalities include both income based instruments - taxes and social security contributions, as well as expenditure instruments such as social transfers. It is estimated that in OECD countries $75 \%$ of the reduction is due to transfers and the rest to direct household taxation (OECD 2012: 3). This fact confirms that the material role in redistribution policy is played by expenditure instruments, although the tax system can supplement the effect.

The aim of the paper is to provide a comparative analysis of the level of Personal Income Tax (PIT) progression in OECD countries and identification of trends in progression in the context of income redistribution.

\section{THE ROLE OF PROGRESSIVE PERSONAL INCOME TAXES IN THE REDUCTION OF INCOME INEQUALITIES}

The impact of individual tax instruments on income dispersion is very diverse. The consumption taxes (especially Value Added Tax and excises on energy) tend to be regressive. They have been described as anti-democratic and anti-family since the XIX century (Gaudemet, Molinier 2000: 496). The regression of VAT and excises is closely linked with the fact that poorer households are characterised by lower propensity to consume, which means that 
they consume a relatively larger share of their income than the better-off. The immovable property tax with the area basis has the same character. (Etel, Dowgier 2013: 14; Głuszak, Marona 2015: 91).

There is a wide consensus that the key tax instrument in redistribution policy is the progressive Personal Income Tax. Its direct and elastic construction enables to adjust the tax burden to individual traits of a given taxpayer and his household.

Progression in personal income taxation was already known in Acient Athens under the reign of Solon (Małecka-Ziemnińska 2012: 143). The theoretical justification of the progressive schedule is the theory of equal sacrifice, formulated by J.S. Mill (1966: 564). Due to progressive PIT schedule, burden of the construction can be linked to individual's capacity to pay (ability-to-pay principle) (Gwiazdowski 2001: 74). A tax is progressive if the average tax rate increases with income or, equivalently, if the marginal tax rate is higher than the average tax rate at a particular level of income (Paturot, Mellbye, Brys 2013: 4). The main premise of the use of such kind of scale is an urgent need for social justice. The progressive scale pursues the principle of equity, which occupies a special place in the discussion of the optimal taxation. The postulate of equitable taxation was formulated by, among others, A. Smith (2007: 572), A. Wagner (Gomułowicz 2001b: 47) and J. Stiglitz (2004: 550-575).

The individual's ability to pay is defined as financial endurance of a taxpayer. It means that it should be possible to a taxpayer to bear the level of tax burden and the tax burden should not cause a taxpayer's resistance (Gomułowicz 2001a: 55). The progression in personal income taxation means that the tax absorbs a larger share of the income of the well-off. As a result, the income disparity after taxation is lower than before taxes.

\section{TRENDS IN PERSONAL INCOME TAX SCHEDULES}

The progressivity of the PIT depends on the level of statutory rates and their relations - in particular the difference between the top and the bottom rate. Another important factor determining the level of progression is the number and width of the tax brackets (Paturot, Mellbye, Brys 2013: 4).

The potential benefits of flat PIT has been widely discussed in the literature (Hall, Rabuska 1998: 79-109; Owens 2013: 679; Homes 2008: 86), the vast majority of OECD countries use the multi-rate formula of the tax. The average number of tax brackets decreased significantly during the 1980s. In 1980 it was common for OECD countries to have 14 tax brackets. The number dropped to 6 by 1990. In 199011 OECD countries had 6 or more brackets, 4 countries had 10 or more brackets and only Iceland used single-rate formula of PIT. By 2010 the reduction trend was continued, but at a slower pace. As a result, the average 
number of tax brackets in OECD countries was reduced to 4. Three Central-East countries (Czech Republic, Estonia and Slovak Republic) adopted flat PIT systems during the period. The trend has been reversed in the 2010s. In 2012 the Slovak Republic resigned from the single-rate formula. Moreover, Finland, Israel, Luxembourg, Spain, Slovenia, Japan, Korea, Mexico and the United States added additional tax bracket(s) to the PIT schedule.

Table 1. Number of Central Personal Income Tax Brackets

\begin{tabular}{|c|c|c|c|c|}
\hline Country / Year & 1990 & 2000 & 2010 & 2014 \\
\hline 1 & 2 & 3 & 4 & 5 \\
\hline Australia & 7 & 4 & 4 & 4 \\
\hline Austria & 5 & 4 & 3 & 3 \\
\hline Belgium & 7 & 7 & 5 & 5 \\
\hline Canada & 3 & 3 & 4 & 4 \\
\hline Chile & n.a. & 6 & 7 & 7 \\
\hline Czech Republic & n.a. & 4 & 1 & 1 \\
\hline Denmark & 3 & 3 & 2 & 2 \\
\hline Estonia & n.a. & 1 & 1 & 1 \\
\hline Finland & 5 & 6 & 4 & 5 \\
\hline France & 12 & 6 & 4 & 4 \\
\hline Germany & 3 & 3 & 4 & 4 \\
\hline Greece & 9 & 5 & 7 & 3 \\
\hline Hungary & 4 & 3 & 2 & 1 \\
\hline Iceland & 1 & 2 & 3 & 3 \\
\hline Ireland & 3 & 2 & 2 & 2 \\
\hline Israel & 5 & 5 & 6 & 7 \\
\hline Italy & 7 & 5 & 5 & 5 \\
\hline Japan & 4 & 4 & 6 & 6 \\
\hline Korea & 4 & 4 & 4 & 5 \\
\hline Luxembourg & 24 & 16 & 16 & 17 \\
\hline Mexico & 6 & 10 & 8 & 11 \\
\hline Netherlands & 3 & 4 & 4 & 2 \\
\hline New Zealand & 3 & 4 & 4 & 3 \\
\hline Norway & 3 & 3 & 3 & 3 \\
\hline Poland & n.a. & 3 & 2 & 2 \\
\hline Portugal & 5 & 5 & 7 & 5 \\
\hline Slovak Republic & n.a. & 7 & 1 & 2 \\
\hline
\end{tabular}


Table 1 (cont.)

\begin{tabular}{|l|c|c|c|c|}
\hline \multicolumn{1}{|c|}{1} & 2 & 3 & 4 & 5 \\
\hline Slovenia & 6 & 3 & 3 & 4 \\
\hline Spain & 16 & 6 & 4 & 7 \\
\hline Sweden & 4 & 2 & 2 & 2 \\
\hline Switzerland & 10 & 10 & 10 & 10 \\
\hline Turkey & 6 & 4 & 4 & 4 \\
\hline United Kingdom & 2 & 3 & 3 & 3 \\
\hline United States & 2 & 5 & 6 & 7 \\
\hline OECD average & $\mathbf{6}$ & $\mathbf{5}$ & $\mathbf{4}$ & $\mathbf{5}$ \\
\hline
\end{tabular}

Source: OECD (2014c).

The changes in the number of tax brackets were closely linked to the changes in the level of statutory tax rates. Despite the fact that the statutory rates are only one factor determining tax burden, their modifications are a powerful policy tool for changing tax burden. In the context of progressivity special role is played by marginal PIT rates. The trend towards lower rates was clear from 2000 to 2009. The level of the rates in the top bracket decreased during the period in 26 out of 33 OECD countries. The strongest reductions took place in France, Germany, Denmark, Norway - the countries where the initial level of the top PIT rate was one of the highest in the Organisation. The trend was accompanied by the reduction in the threshold where the top rates apply (Table 2). In contrast, at the same time, OECD countries diverged in their policies regarding changes in their bottom statutory rates.

Table 2. The level of the top statutory Personal Income Tax rates in OECD countries

\begin{tabular}{|l|c|c|c|c|c|c|c|c|c|c|c|c|}
\hline \multirow{2}{*}{$\begin{array}{c}\text { Country / } \\
\text { Year }\end{array}$} & \multicolumn{2}{|c|}{2000} & \multicolumn{2}{c|}{2004} & \multicolumn{2}{c|}{2008} & \multicolumn{2}{c|}{2010} & \multicolumn{2}{|c|}{2012} & \multicolumn{2}{c|}{2014} \\
\cline { 2 - 15 } & t.t.r. & thr. & t.t.r. & thr. & t.t.r. & thr. & t.t.r. & thr. & t.t.r. & thr. & t.t.r. & thr. \\
\hline 1 & 2 & 3 & 4 & 5 & 6 & 7 & 8 & 9 & 10 & 11 & 12 & 13 \\
\hline Australia & 48.50 & 1.21 & 48.50 & 1.24 & 46.50 & 2.49 & 46.50 & 2.70 & 47.50 & 2.45 & 46.50 & 2.26 \\
\hline Austria & 50.00 & 2.33 & 50.00 & 2.07 & 50.00 & 1.87 & 50.00 & 2.10 & 50.00 & 1.99 & 50.00 & 1.92 \\
\hline Belgium & 60.50 & 2.33 & 53.50 & 1.05 & 53.70 & 1.01 & 53.70 & 0.99 & 53.70 & 0.99 & 53.70 & 1.02 \\
\hline Canada & 47.86 & 2.10 & 46.41 & 2.97 & 46.41 & 2.87 & 46.41 & 2.86 & 47.97 & 10.65 & 49.53 & 4.45 \\
\hline Chile & 45.00 & 16.33 & 40.00 & 19.04 & 40.00 & 18.26 & 40.00 & 14.03 & 40.00 & 13.40 & 40.00 & 12.76 \\
\hline $\begin{array}{l}\text { Czech } \\
\text { Republic }\end{array}$ & 32.00 & 2.46 & 32.00 & 2.02 & 15.00 & 0.45 & 15.00 & 0.43 & 15.00 & 0.41 & 15.00 & 0.40 \\
\hline Denmark & 59.70 & 1.05 & 59.73 & 1.05 & 59.73 & 1.02 & 52.24 & 1.13 & 60.23 & 1.08 & 60.42 & 1.23 \\
\hline Estonia & 26.00 & 0.16 & 26.00 & 0.19 & 21.00 & 0.17 & 21.00 & 0.18 & 21.00 & 0.16 & 21.00 & 0.14 \\
\hline
\end{tabular}


Table 2 (cont.)

\begin{tabular}{|c|c|c|c|c|c|c|c|c|c|c|c|c|}
\hline 1 & 2 & 3 & 4 & 5 & 6 & 7 & 8 & 9 & 10 & 11 & 12 & 13 \\
\hline Finland & .17 & 2.15 & 2.12 & 1.93 & 50.05 & 1.77 & 48.98 & 1.81 & 49.00 & 1.83 & 51.49 & 2.5 \\
\hline ance & .27 & 2.81 & 3.36 & 2.75 & 45.78 & 2.79 & 46.73 & 2.76 & 54.41 & 15.42 & 54.50 & 14.9 \\
\hline ermany & .81 & 1.79 & 7.48 & 1.45 & 47.48 & 6.21 & 47.48 & 6.19 & 47.48 & 5.85 & 47.48 & 5.6 \\
\hline reece & 5.00 & 3.61 & 40.00 & 1.29 & 40.00 & 3.75 & 45.00 & 4.93 & 49.00 & 5.39 & 46.00 & 0.0 \\
\hline ungar & .00 & 0.92 & 8.00 & 0.89 & 36.00 & 0.73 & 32.00 & 1.57 & 16.00 & 0.85 & 16.00 & 0.0 \\
\hline 19110 & 5.37 & 1.21 & 2.58 & 1.16 & 35.72 & 0.22 & 46.12 & 1.55 & 46.24 & 1.44 & 46.24 & 1.4 \\
\hline , & .00 & 0.98 & 42.00 & 1.00 & 41.00 & 1.09 & 47.00 & 5.42 & 48.00 & 1.01 & 48.00 & 0.9 \\
\hline & .00 & 2.33 & 9.00 & 3.97 & 47.00 & 3.65 & 45.00 & 3.88 & 48.00 & 3.91 & 50.00 & 6.2 \\
\hline & 0 & 3.57 & 6.10 & 3.27 & 44.90 & 3.10 & 45.20 & 2.95 & 48.55 & 10.19 & 49.13 & 9.8 \\
\hline & 00 & 4.54 & 0.00 & 4.54 & 50.00 & 4.51 & 50.00 & 4.73 & 50.00 & 4.63 & ד. & $4.5^{7}$ \\
\hline & .00 & 5.41 & 9.60 & 3.83 & 38.50 & 3.31 & 38.50 & 3.01 & 41.80 & 8.86 & 41.80 & 44 \\
\hline & 47.15 & 2.09 & 38.95 & 1.01 & 38.95 & 0.92 & 38.95 & 0.94 & 41.34 & 3.15 & 43.60 & 3.0 \\
\hline Mexi & 40.00 & 49.75 & 33.00 & 1.70 & 28.00 & 4.86 & 30.00 & 4.53 & 30.00 & 4.17 & 35.00 & 29.4 \\
\hline Netherl & 60.00 & 1.76 & 52.00 & 1.38 & 52.00 & 1.25 & 52.00 & 1.19 & 52.00 & 1.16 & 52.00 & 1.2 \\
\hline New Zealand & 8.00 & 1.72 & 39.00 & 1.55 & 39.00 & 1.57 & 35.50 & 1.46 & 33.00 & 1.37 & 33.00 & 1.0 \\
\hline Jiway & 7.50 & 2.56 & 47.50 & 2.48 & 40.00 & 1.54 & 40.00 & 1.57 & 40.00 & 1.58 & 39.00 & 1.5 \\
\hline oland & 0.00 & 3.55 & 40.00 & 3.28 & 40.00 & 2.93 & 32.00 & 2.74 & 32.00 & 2.50 & 32.00 & 2.38 \\
\hline$P$ & .00 & 3.38 & 0.00 & 4.37 & 42.00 & 4.51 & 45.88 & 10.19 & 49.00 & 10.11 & 56.50 & 16.1 \\
\hline & 42.00 & 7.95 & 19.00 & 0.49 & 19.00 & 0.43 & 19.00 & 0.50 & 19.00 & 0.43 & 25.00 & 3.9 \\
\hline & .00 & 4.28 & 50.00 & 4.27 & 41.00 & 1.41 & 41.00 & 1.38 & 41.00 & 1.38 & 50.00 & 5.3 \\
\hline & 8.00 & 4.31 & 45.00 & 2.64 & 43.00 & 2.51 & 43.00 & 2.36 & 52.00 & 11.78 & 52.00 & 11.6 \\
\hline wed & 5.38 & 1.51 & 56.51 & 1.50 & 56.44 & 1.44 & 56.56 & 1.48 & 56.60 & 1.51 & 56.86 & 1.5 \\
\hline Switze & 43.75 & 3.62 & 42.06 & 3.33 & 41.67 & 3.32 & 41.67 & 3.24 & 41.67 & 3.36 & 41.67 & 3.3 \\
\hline Turkey & 40.60 & 11.46 & 40.60 & 10.39 & 35.60 & 2.73 & 35.66 & 4.37 & 35.66 & 4.22 & 35.76 & 3.8 \\
\hline $\begin{array}{l}\text { United } \\
\text { Kingdom }\end{array}$ & 40.00 & 1.32 & 40.00 & 1.23 & 40.00 & 1.22 & 50.00 & 4.37 & 50.00 & 4.30 & 45.00 & 4.2 \\
\hline & 46.65 & 8.81 & 41.50 & 8.78 & 41.85 & 8.44 & 41.85 & 8.39 & 41.78 & 8.34 & 46.25 & 8.2 \\
\hline OECD & 46.52 & 4.86 & 43.57 & 3.06 & 41.39 & 2.89 & 41.76 & 3.29 & 42.62 & 4.41 & 43.57 & 5.22 \\
\hline
\end{tabular}

t.t.r. - total statutory tax (PIT) rate

thr. - threshold at which the top rate is applied - expressed as a multiple of the average wage

Source: OECD (2014d).

After 2011 the trend has been reversed - the rates have risen, but have not reached their level from the year 2000. The change can be plausibly attributed to the effect of the economic and financial crisis (European Commission 2013: 33). 
Out of different kinds of tax expenditures the key role in narrowing income disparities is played by the provisions which exempt an initial level of income from PIT - in the form of exceptions, reductions or credits (Paturot, Mellbye, Brys 2013: 4). The instrument has increased the progressivity of Personal Income Taxes, particularly in the countries with flat PIT systems. Its justification is that a minimum of marked income should be free of tax because it is spent on necessities (Hybka 2005: 53). What's more, it is useful in reducing poverty.

Table 3. Central level Zero-Rate Brackets, Basic Allowances and Basic Tax Credits (as a Percentage of the Average Wage) in OECD countries in 2000 and 2010 year

\begin{tabular}{|c|c|c|c|c|}
\hline \multirow[b]{2}{*}{ Country } & \multicolumn{2}{|c|}{2000} & \multicolumn{2}{|l|}{2010} \\
\hline & measure & $\begin{array}{c}\% \text { of average } \\
\text { wage }\end{array}$ & measure & $\begin{array}{c}\% \text { of average } \\
\text { wage }\end{array}$ \\
\hline 1 & 2 & 3 & 4 & 5 \\
\hline \multirow{2}{*}{ Australia } & zero-rate bracket & 15 & zero-rate bracket & 15 \\
\hline & wastable tax credit (i) & 2 & wastable tax credit (i) & 13 \\
\hline Austria & zero-rate bracket & 12 & zero-rate bracket & 29 \\
\hline Belgium & basic allowance & 16 & basic allowance & 15 \\
\hline Canada & wastable tax credit & 19 & wastable tax credit & 23 \\
\hline Chile & basic allowance & 120 & basic allowance & 111 \\
\hline Czech Republic & basic allowance & 22 & wastable credit & 43 \\
\hline Denmark & wastable tax credit & 12 & wastable tax credit & 11 \\
\hline Estonia & basic allowance & 16 & basic allowance & 18 \\
\hline Finland & zero-rate bracket & 30 & zero-rate bracket & 38 \\
\hline France & zero-rate bracket & 15 & zero-rate bracket & 17 \\
\hline Germany & zero-rate bracket & 20 & zero-rate bracket & 19 \\
\hline Greece & zero-rate bracket & 71 & zero-rate bracket & 59 \\
\hline Hungary & - & - & - & - \\
\hline Iceland & wastable tax credit & 41 & wastable tax credit & 42 \\
\hline \multirow{2}{*}{ Ireland } & \multirow{2}{*}{ wastable tax credit } & \multirow{2}{*}{21} & wastable tax credit & 23 \\
\hline & & & basic allowance $(\mathrm{t})$ & 13 \\
\hline Israel & wastable tax credit & & wastable tax credit & \\
\hline Italy & - & - & wastable tax credit & 28 \\
\hline Japan & basic allowance & 8 & basic allowance & 8 \\
\hline Korea & basic allowance & 3 & basic allowance & 4 \\
\hline Luxembourg & zero-rate bracket & 19 & zero-rate bracket & 23 \\
\hline Mexico & - & - & - & - \\
\hline Netherlands & basic allowance & 13 & wastable tax credit & 13 \\
\hline New Zealand & - & - & - & \\
\hline Norway & basic allowance (i) & 20 & basic allowance (i) & 16 \\
\hline Poland & wastable tax credit & 10 & wastable tax credit & 9 \\
\hline \multirow{2}{*}{ Portugal } & \multirow{2}{*}{ wastable tax credit } & \multirow{2}{*}{12} & wastable tax credit & 14 \\
\hline & & & basic allowance & 24 \\
\hline Slovak Republic & basic allowance & 25 & basic allowance (t) & 43 \\
\hline
\end{tabular}


Table 3 (cont.)

\begin{tabular}{|l|l|r|l|c|}
\hline \multicolumn{1}{|c|}{1} & \multicolumn{1}{c|}{2} & \multicolumn{1}{c|}{4} & 5 \\
\hline Slovenia & basic allowance & 12 & basic allowance $(\mathrm{t})$ & 36 \\
\hline Spain & basic allowance & 19 & basic allowance & 21 \\
\hline \multirow{3}{*}{ Sweden } & zero-rate bracket & 88 & zero-rate bracket & 101 \\
\cline { 2 - 5 } & basic allowance (i) (t) & 3 & \multirow{2}{*}{ basic allowance (i) (t) } & 5 \\
\cline { 2 - 5 } & non-wastable tax credit (t) & 3 & zero-rate bracket & 18 \\
\hline Switzerland & zero-rate bracket & 25 & - & - \\
\hline Turkey & basic allowance & 4 & & 19 \\
\hline United Kingdom & basic allowance & 18 & basic allowance $(\mathrm{t})$ & 13 \\
\hline United States & basic allowance & 22 & basic allowance & \\
\hline
\end{tabular}

(t) amount is tapered with income

(i) amount increases with income (up to a limit)

Source: Torres, Mellbye, Brys (2012): 21.

The instrument takes on three possible forms. At the moment a comparable number of countries use the zero-rate tax bracket (9), the basic personal allowance (14) and the wastable tax credit (11). The vast majority of OECD countries use the instrument. In Australia, Ireland, Sweden, Norway, the United Kingdom, Slovak Republic and Slovenia its redistributive effect is strengthened by the fact that the amount of exempted minimum changes according to the level of income. The only OECD country which withdrew the instrument is Hungary.

In the period 2000-2010 a significant (at least 2 p.p.) increase in the value of basic exemptions as a share of the average wage took place in 12 OECD countries. At the same time the value did not considerably changed in 13 counties and it decreased in 6 countries.

From a redistributional perspective it is important to link the level of the exempted income with the minimum subsistence figure in a given country. Meanwhile in Korea, Japan and Poland it is lower that $10 \%$ of the average wage. The relation between the level of exempted income and the average wage vary substantially in OECD countries.

In 2014 all but one OECD countries differentiated the tax burden from the level of income (Table 4). The difference between average income tax rate at $167 \%$ average earnings and the same rate at $67 \%$ average earnings (on the assumption that the person is single and doesn't have any children) is the highest (23 p.p.) in the Netherlands. Its value exceeds 15 p.p. in Ireland, Luxembourg, Greece, Sweden, Portugal and Italy. In contrast, in Hungary the tax burden does not change with the level of income. In Chile and Poland the reduction is marginal - the difference in the two average income rates described above is lower than 2 p.p. 


\begin{tabular}{|c|c|c|c|c|c|c|c|c|c|c|c|c|c|c|c|c|c|c|c|c|c|}
\hline 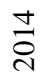 & 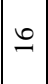 & $\mid \begin{array}{l}0 \\
\\
=\end{array}$ & 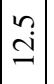 & 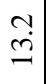 & $\stackrel{?}{0}$ & $\hat{o}$ & $\vec{r}$ & $\tilde{a}$ & $\begin{array}{l}0 \\
i\end{array}$ & $\begin{array}{l}\infty \\
\ddot{2}\end{array}$ & $\infty$ & $\ddot{n}$ & $\begin{array}{l}n \\
n \\
n\end{array}$ & $\ddot{0}$ & 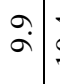 & $\begin{array}{l}\ddot{2} \\
\stackrel{2}{2}=\end{array}$ & $\stackrel{0}{=}$ & $\begin{array}{ll}0 \\
\ddot{n}\end{array}$ & $\overrightarrow{6}$ & $\begin{array}{l}\infty \\
0 \\
0\end{array}$ & $\begin{array}{l}\infty \\
\dot{n} \\
n\end{array}$ \\
\hline 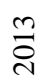 & 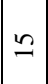 & $\stackrel{9}{=}$ & $\begin{array}{l}0 \\
\mathbf{I} \\
=\end{array}$ & $\stackrel{\sim}{2}$ & $\stackrel{\Upsilon}{0}$ & $\stackrel{\infty}{0}$ & $\stackrel{m}{r}$ & $\tilde{a}$ & $\begin{array}{l}\infty \\
i\end{array}$ & $\begin{array}{l}\infty \\
\dot{2}\end{array}$ & $\begin{array}{c}1 \\
\infty \\
\infty\end{array}$ & $\begin{array}{l}n \\
\ddot{2}\end{array}$ & $\begin{array}{l}0 \\
\stackrel{0}{2}\end{array}$ & $\because$ & $\hat{a}$ & $\stackrel{9}{2}$ & $\stackrel{n}{=}=$ & $\underset{0}{0}$ & $\stackrel{0}{0}$ & $\stackrel{?}{\sim}$ & $\stackrel{a}{a}$ \\
\hline 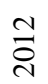 & $\Xi$ & $\underset{\nearrow}{\simeq}$ & $\hat{\mathrm{\jmath}}$ & $\stackrel{0}{\ddot{g}}$ & $\stackrel{\Upsilon}{0}$ & $\ddot{0}$ & $\stackrel{m}{r}$ & $\stackrel{0}{\circ}$ & $\stackrel{0}{r}$ & $\stackrel{\sim}{ \pm}$ & $\begin{array}{c}1 \\
\infty \\
\infty\end{array}$ & $\ddot{n}$ & $\stackrel{\vartheta}{0}$ & $\vec{i}$ & $\overrightarrow{0}$ & $\stackrel{m}{2}=$ & $\begin{array}{ll}0 \\
= & \end{array}$ & $\exists$ & $\overrightarrow{6}$ & $?$ & $\begin{array}{l}\ddot{2} \\
\therefore\end{array}$ \\
\hline$\overline{\vec{D}}$ & 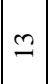 & 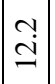 & \begin{tabular}{|c|}
0 \\
$\ddot{2}$
\end{tabular} & 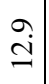 & $\stackrel{\Upsilon}{\stackrel{0}{0}}$ & $\tilde{o}$ & $\stackrel{n}{r}$ & $\hat{\sigma}$ & $\vec{m}$ & $\ddot{\ddot{g}}$ & $\vec{\infty}$ & $\begin{array}{l}0 \\
\ddot{2}\end{array} \mid$ & $\exists$ & $\mid \begin{array}{ll}2 \\
\infty \\
\infty\end{array}$ & $\ddot{0}:$ & $\stackrel{2}{\varrho}=$ & $\stackrel{0}{=}$ & $\exists$ & $\begin{array}{l}0 \\
\dot{0}\end{array}$ & $\vec{r}$ & $\stackrel{a}{n}$ \\
\hline$\stackrel{0}{\stackrel{0}{\circ}}$ & $\simeq$ & 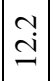 & $\begin{array}{l}\tilde{2} \\
\ddot{2}\end{array}$ & $\stackrel{0}{\ddot{\theta}}$ & $\stackrel{\Upsilon}{0}$ & ó & $\stackrel{2}{2}$ & $\stackrel{\infty}{a}$ & $\stackrel{m}{m}$ & $\begin{array}{l}\infty \\
\ddot{2}\end{array}$ & $\begin{array}{c}0 \\
\infty\end{array}$ & $\begin{array}{l}\dot{\theta} \\
\ddot{2}\end{array}$ & $\begin{array}{l}0 \\
\stackrel{1}{J}\end{array}$ & $\stackrel{\rho}{=}$ & $\stackrel{0}{0}$ & $\stackrel{0}{=}$ & $\stackrel{0}{\check{I}}$ & $=$ & $\stackrel{\vartheta}{\grave{n}}$ & 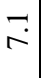 & $\begin{array}{l}\ddot{\bullet} \\
\stackrel{\sim}{2}\end{array}$ \\
\hline ஓे & $=$ & 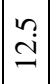 & $\begin{array}{c}m \\
\stackrel{2}{2}\end{array}$ & $\begin{array}{l}\tilde{y} \\
\ddot{2}\end{array}$ & $\hat{a}$ & $\ddot{0}$ & $\frac{9}{r}$ & $\stackrel{\mathrm{g}}{\mathrm{j}}$ & $\stackrel{+}{\dot{r}}$ & $\begin{array}{l}n \\
\ddot{2}\end{array}$ & $\begin{array}{l}0 \\
\infty \\
\infty\end{array}$ & $\underline{\ddot{g}}$ & 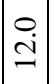 & $\mid \begin{array}{l}\infty \\
\underline{n}\end{array}$ & $\begin{array}{l}a \\
\infty\end{array}$ & $\stackrel{0}{0}$ & \begin{tabular}{lll}
$\stackrel{0}{1}$ & \multirow{2}{c}{} &
\end{tabular} & 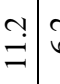 & 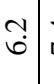 & $\stackrel{⿱}{\sim}$ & $\stackrel{+}{\check{L}}$ \\
\hline$\stackrel{\infty}{\stackrel{\sim}{8}}$ & 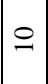 & $\stackrel{+}{\Xi}$ & $\hat{\mathrm{c}}$ & $\vec{m}$ & 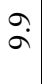 & $\stackrel{0}{0}$ & $\underset{\infty}{\infty}$ & $\stackrel{+}{\ddot{\theta}}$ & $\stackrel{\sim}{m}$ & $\stackrel{m}{2}$ & $\begin{array}{c}0 \\
\infty\end{array}$ & $\begin{array}{l}\infty \\
\ddot{2}\end{array}$ & $\begin{array}{l}0 \\
\ddot{g}\end{array}$ & $\underset{1}{0}$ & $\tilde{6}$ & 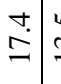 & $\stackrel{n}{\check{c}}$ & $\underset{1}{=}$ & $\begin{array}{lll}n \\
n\end{array}$ & $\stackrel{\circ}{r}$ & $\begin{array}{l}0 \\
\ddot{n}\end{array}$ \\
\hline ఠ్ & $a$ & $\begin{array}{l}0 \\
\dot{a}\end{array}$ & $\hat{\mathrm{a}}$ & $\begin{array}{l}0 \\
\ddot{2}\end{array}$ & $\tilde{a}$ & $\stackrel{0}{0}$ & $\begin{array}{l}m \\
\infty\end{array}$ & $\overrightarrow{\ddot{m}}$ & $\stackrel{+}{\ddot{r}}$ & $\begin{array}{l}0 \\
\ddot{2}\end{array}$ & $\begin{array}{c}0 \\
\infty\end{array}$ & $\begin{array}{l}\infty \\
\ddot{2}\end{array}$ & $\ddot{\ddot{g}}$ & $\stackrel{+}{\stackrel{5}{\Sigma}}$ & $\begin{array}{l}\infty \\
0\end{array}$ & $\begin{array}{l}\Delta \\
\check{\Sigma}\end{array}$ & $\stackrel{\circ}{ \pm}$ & $\stackrel{c}{c}$ & "n & $\stackrel{\infty}{\sim}$ & $\begin{array}{l}g \\
\dot{J}\end{array}$ \\
\hline ஜㅇ & $\infty$ & $\stackrel{n}{0}$ & $\vec{m}$ & $\stackrel{0}{\ddot{g}}$ & $\stackrel{\nabla}{\circ}$ & $\stackrel{\circ}{0}$ & $\vec{\infty}$ & $\stackrel{0}{\ddot{g}}$ & $\stackrel{r}{+}$ & 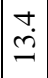 & $\stackrel{9}{r}$ & $\dot{\ddot{g}}$ & 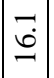 & $\begin{array}{l}0 \\
\infty \\
\infty\end{array}$ & $\begin{array}{l}\infty \\
0\end{array}$ & 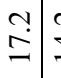 & $\stackrel{\sim}{\stackrel{I}{ \pm}}$ & $\hat{0}$ & \begin{tabular}{l|l}
0 & \\
$\dot{0}$ & 1
\end{tabular} & $\stackrel{n}{r}$ & $\begin{array}{l}0 \\
\ddot{n}\end{array}$ \\
\hline$\stackrel{\overbrace{}}{ి}$ & $r$ & $\begin{array}{l}0 \\
= \\
=\end{array}$ & $\stackrel{+}{\stackrel{2}{2}}$ & 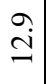 & $\stackrel{\forall}{\circ}$ & $\stackrel{0}{0}$ & $\begin{array}{l}0 \\
\dot{n}\end{array}$ & $\stackrel{\mathrm{i}}{\mathrm{j}}$ & $\stackrel{+}{+}$ & $\stackrel{m}{m}$ & $\stackrel{2}{r}$ & $\underline{\ddot{g}}$ & 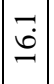 & $\stackrel{m}{2}$ & $\ddot{a}$ & 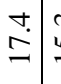 & $\stackrel{m}{2} \stackrel{9}{2}$ & \begin{tabular}{l|l}
$\infty$ & \\
$\stackrel{0}{0}$ &
\end{tabular} & $\begin{array}{l}\qquad \\
\dot{r}\end{array}$ & $\vec{r}$ & $=$ \\
\hline ষ্ণ & 0 & 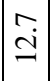 & $\begin{array}{l}0 \\
\ddot{2}\end{array}$ & $\begin{array}{l}0 \\
\ddot{2}\end{array}$ & $\stackrel{\infty}{\infty}$ & $\stackrel{0}{0}$ & $\begin{array}{l}\dot{n} \\
\dot{n}\end{array}$ & $\stackrel{0}{\ddot{g}}$ & $\stackrel{+}{+}$ & $\stackrel{+}{\dot{2}}$ & 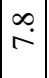 & $\begin{array}{l}0 \\
\ddot{2}\end{array}$ & $\begin{array}{l}\tilde{n} \\
\ddot{n}\end{array}$ & $\begin{array}{l}n \\
\infty\end{array}$ & $\underset{\simeq}{\simeq}$ & $\begin{array}{l}0 \\
\Sigma\end{array}$ & $\begin{array}{l}0 \\
\dot{\imath}\end{array}$ & \begin{tabular}{l|l}
0 & $v$ \\
$=$ & $v$
\end{tabular} & $\begin{array}{l}n \\
n \\
n\end{array}$ & $\vec{r}$ & $=$ \\
\hline §ิ) & in & $\stackrel{n}{c}$ & $\begin{array}{l}\infty \\
\stackrel{\sim}{c}\end{array}$ & $\stackrel{a}{\beth}$ & $\vec{a}$ & $\stackrel{\circ}{\circ}$ & N̦ & $\overrightarrow{\ddot{g}}$ & $\stackrel{m}{m}$ & $\stackrel{m}{2}$ & $\stackrel{9}{r}$ & $\mid \begin{array}{l}a \\
\check{2}\end{array}$ & $\stackrel{n}{ \pm}$ & $\begin{array}{l}a \\
\infty \\
-\end{array}$ & $\begin{array}{l}0 \\
\dot{\sim}\end{array}$ & ڤેc & $\stackrel{r}{\underline{m}}=$ & \begin{tabular}{l|l}
0 & $v$ \\
$=$ & $v$
\end{tabular} & $n$ & $\hat{0}$ & $\begin{array}{l}n \\
\\
n\end{array}$ \\
\hline §ิ & $\nabla$ & 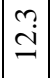 & 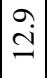 & $\begin{array}{l}\infty \\
\stackrel{\sim}{\sim} \\
-\end{array}$ & $\stackrel{\circ}{\circ}$ & $\stackrel{\circ}{\circ}$ & $\begin{array}{c}0 \\
\dot{n}\end{array}$ & $\overrightarrow{\ddot{g}}$ & $\underset{\text { ri }}{0}$ & $\stackrel{m}{m}$ & $\vec{\infty}$ & $\mid \begin{array}{l}\infty \\
\ddot{1}\end{array}$ & $\stackrel{n}{a}$ & $\begin{array}{l}0 \\
\stackrel{\cos }{2}\end{array}$ & $\stackrel{a}{\varrho}$ & $\ddot{\sim}$ & $\stackrel{m}{ \pm}$ & $\sigma^{a} \mid v$ & $n$ & ชુ & $\begin{array}{l}n \\
\end{array}$ \\
\hline 호 & $m$ & 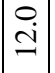 & $\begin{array}{l}0 \\
\ddot{2}\end{array}$ & $\stackrel{0}{\ddot{g}}$ & $\vec{a}$ & $n$ & $\begin{array}{l}\infty \\
\dot{\forall}\end{array}$ & $\stackrel{m}{2}$ & 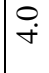 & $\stackrel{?}{2}$ & $\grave{\infty}$ & $\begin{array}{l}0 \\
\ddot{2}\end{array}$ & $\stackrel{.}{\Xi}$ & $\overrightarrow{\mathrm{d}}$ & $\ddot{0}$ & 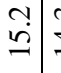 & $\stackrel{?}{ \pm}$ & \begin{tabular}{l|l}
$\infty$ & v \\
$\sigma$ & v
\end{tabular} & $\stackrel{i}{i}$ & $\stackrel{\nabla}{0}$ & $\ddot{0}$ \\
\hline 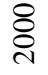 & $N$ & $\begin{array}{l}\infty \\
\stackrel{\vartheta}{-}\end{array}$ & $\begin{array}{l}\infty \\
\stackrel{\jmath}{\beth}\end{array}$ & 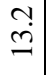 & $\stackrel{n}{\varrho}$ & $n$ & $\stackrel{F}{F}$ & $\vec{\Omega}$ & $\stackrel{0}{0}$ & $\overrightarrow{\ddot{2}}$ & $\grave{\infty}$ & 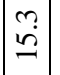 & $\stackrel{n}{=}$ & $\hat{\mathrm{i}}$ & $\begin{array}{l}0 \\
0 \\
0\end{array}$ & $\begin{array}{l}0 \\
\stackrel{0}{L}\end{array}$ & $\stackrel{\sim}{ \pm}$ & $\overrightarrow{0}:$ & $\vec{n}$ & iे & $\begin{array}{l}0 \\
0 \\
0\end{array}$ \\
\hline 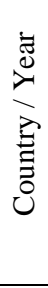 & - & 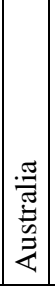 & 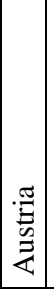 & 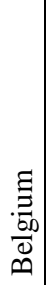 & & $\frac{0}{\exists}$ & 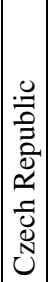 & 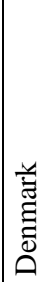 & $\begin{array}{l}\cdot \frac{\pi}{Z} \\
\frac{0}{\tilde{n}} \\
\text { rn }\end{array}$ & 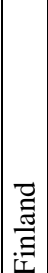 & 苞 & 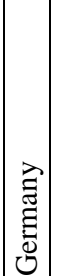 & 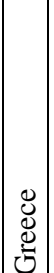 & 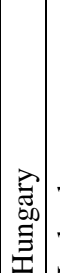 & $\begin{array}{l}\vec{\Xi} \\
\stackrel{\Xi}{\mathbb{E}} \\
\end{array}$ & 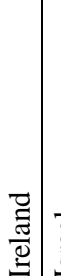 & 串 & $\underset{\Xi}{\Xi}$ & 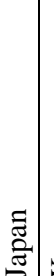 & $\begin{array}{l}\mathscr{d} \\
\stackrel{0}{0} \\
\square\end{array}$ & 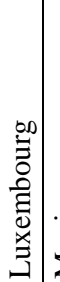 \\
\hline
\end{tabular}




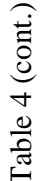

\begin{tabular}{|c|c|c|c|c|c|c|c|c|c|c|c|c|c|c|}
\hline 으 & तె & $\hat{a}$ & $\stackrel{\infty}{\infty}$ & I & $\stackrel{n}{n}$ & ָৃ & $\stackrel{0}{\circ}$ & $\stackrel{?}{0}$ & $\begin{array}{l}0 \\
i \\
\end{array}$ & $\underset{\infty}{\infty}$ & ర0 & $\stackrel{0}{=}$ & $\stackrel{\infty}{\infty}$ & $\stackrel{?}{0}$ \\
\hline$\because$ & $\begin{array}{l}0 \\
\text { ì }\end{array}$ & $\tilde{a}$ & $\ddot{0}$ & . & $\stackrel{n}{n}$ & ?ִ & $\stackrel{9}{\infty}$ & $\stackrel{+}{\circ}$ & in & $\stackrel{9}{r}$ & $\underset{0}{0}$ & $\stackrel{i}{0}$ & $\stackrel{\infty}{\infty}$ & $\stackrel{?}{0}$ \\
\hline$\Xi$ & $\begin{array}{l}\stackrel{\circ}{+} \\
\stackrel{+}{N}\end{array}$ & ח? & $\ddot{0}$ & $\stackrel{\infty}{-}$ & $\begin{array}{l}0 \\
\sim\end{array}$ & $\stackrel{\nabla}{\nabla}$ & $\stackrel{+}{\circ}$ & $\stackrel{n}{0}$ & $\begin{array}{l}\vec{v} \\
\ddot{\nu}\end{array}$ & $\stackrel{9}{r}$ & $\stackrel{+}{\circ}$ & $\stackrel{0}{0}$ & $\stackrel{\ominus}{\infty}$ & $\stackrel{\sim}{\varrho}$ \\
\hline 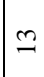 & $\stackrel{m}{\tilde{d}}$ & $\stackrel{\circ}{\circ}$ & $\ddot{0}$ & $\stackrel{\infty}{-}$ & $\stackrel{n}{n}$ & $\stackrel{+}{\circ}$ & $\begin{array}{l}0 \\
0\end{array}$ & ๙n & $\stackrel{n}{n}$ & $\stackrel{\infty}{\sim}$ & $\stackrel{+}{0}$ & $\stackrel{\circ}{\circ}$ & $\stackrel{\circ}{\circ}$ & $\stackrel{?}{?}$ \\
\hline I & $\vec{d}$ & $\vec{a}$ & $\ddot{0}$ & $\stackrel{\circ}{\circ}$ & $\begin{array}{l}\dot{\sim} \\
\stackrel{\sim}{*}\end{array}$ & $\stackrel{+}{\sim}$ & $\stackrel{+}{\circ}$ & ণे & $\begin{array}{l}\infty \\
i\end{array}$ & $\stackrel{\Omega}{r}$ & ?ִ & $\vec{\infty}$ & $\stackrel{\infty}{a}$ & $\stackrel{+}{\circ}$ \\
\hline 二 & $\begin{array}{r}\dot{\lambda} \\
\dot{\lambda}\end{array}$ & $a$ & $\ddot{0}$ & $\stackrel{\circ}{\text { ¿ }}$ & $\begin{array}{l}\infty \\
\stackrel{\sim}{J}\end{array}$ & $\stackrel{\circ}{\circ}$ & $\underset{\infty}{\infty}$ & $\stackrel{\infty}{0}$ & $\stackrel{n}{2}$ & $\stackrel{9}{r}$ & $\underset{0}{0}$ & N & $\stackrel{\infty}{a}$ & $\stackrel{n}{?}$ \\
\hline 으 & $\stackrel{\widetilde{N}}{\mathrm{~N}}$ & $\underset{\infty}{\infty}$ & $\ddot{0}$ & $\begin{array}{l}\infty \\
\sim\end{array}$ & 금 & $\stackrel{\nabla}{\forall}$ & $\stackrel{\infty}{\infty}$ & $\hat{\theta}$ & $\ddot{n}$ & $\stackrel{a}{r}$ & o & $\underset{\infty}{\infty}$ & $\stackrel{\circ}{\circ}$ & $\because$ \\
\hline
\end{tabular}

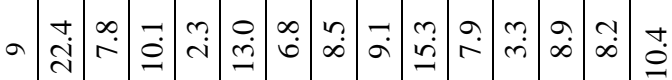

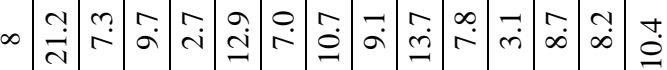

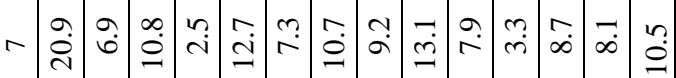

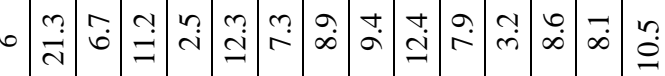

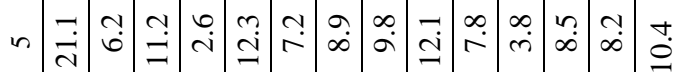

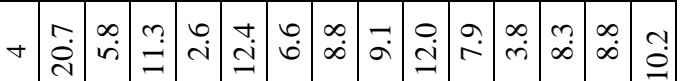

守

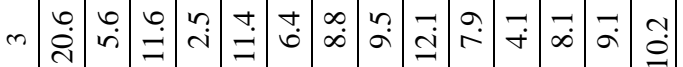

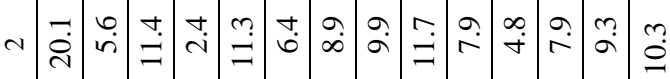

ర్ల

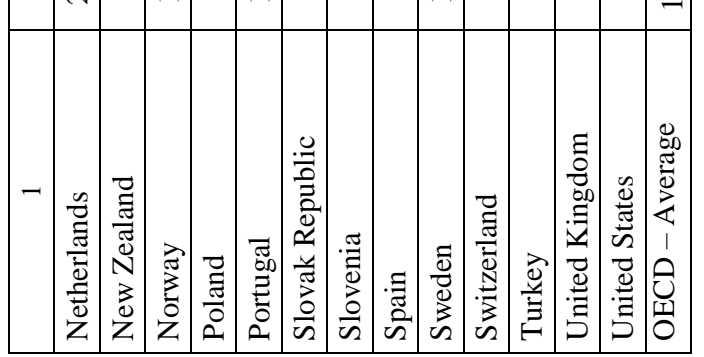


The only OECD country that currently does not differentiate the tax burden - Hungary - has a single-rate PIT and does not use the provisions that exempt an initial level of income from tax. In other countries with flat tax regimes (Estonia, Czech Republic) the scale of the reduction does not exceed the OECD-wide average. Despite the described fact, in Chile - a country with a 7-brackets PIT system - the difference between average income tax rates at $167 \%$ and $67 \%$ of average wage is marginal. Poland also has a multiple rates PIT schedule and the scale of differentiation of tax burden is also marginal.

In the early 2000s the majority of OECD countries (16) tended to make their Personal Income Tax schedules more neutral. As a result, the redistributional effect of PIT was diminished. After the Great Recession of 2008-2009 the trend was reversed. In 19 OECD countries the difference between average tax rates on high and low incomes widened. The main reason for that was the urgent need for fiscal consolidation. Under the circumstances the governments of these countries decided to shift the tax burden to the better-off.

\section{SUMMARY / CONCLUSION}

The Progressive Personal Income Tax is the most widespread tax instrument used in narrowing income disparities. Nowadays all but one OECD countries reduce tax burden with the level of income, although the scale of the reduction varies substantially. The redistributional properties of PIT are determined by the level of statutory rates (especially the top and the bottom rate and their relations), the number of tax brackets and provisions that reduce tax liability with the special importance of amount of income that does not cause the tax liability. The character of the PIT scale does not prejudge the redistribution properties of the construction. The level of the top PIT rate also plays an important role. The correlation between the scale of the tax burden and the top PIT rate measured by Pearson index seems to be significant. Another key factor is the presence and the level of the amount of income that is exempted from PIT (in the form of zero-rate tax bracket, personal allowance or tax credit). The instrument exists in tax systems of all OECD countries with the exception of Hungary, Mexico and Turkey. There is no clear trend in the level of PIT progressivity in OECD. The difference between the average income tax rate at $167 \%$ average earnings and the average income tax rate at $67 \%$ during 2000 2014 has fluctuated about 10 p.p. At the same time the level of income disparities has widened. OECD countries face an urgent problem to counteract the phenomenon. They should consider widening the use of PIT to mitigate income inequalities. 


\section{BIBLIOGRAPHY}

Etel L., Dowgier R. (2013), Podatki i optaty lokalne. Czas na zmiany, Wydawnictwo Temida 2, Białystok.

European Commission (2013), Taxation trends in the European Union. Data for the EU Member States, Iceland and Norway, Eurostat.

Gaudemet P.M., Molinier P. (2000), Finanse publiczne, Polskie Wydawnictwo Ekonomiczne, Warszawa.

Galbraith J.K. (1996), The Good Society. The Humane Agenda, Hougson Mifelin Company, Boston New York.

Głuszak M., Marona B. (2015), Podatek katastralny. Ekonomiczne uwarunkowania reformy opodatkowania nieruchomości, Poltext, Warszawa.

Gomułowicz A. (2001), Zasada sprawiedliwości podatkowej, Dom Wydawniczy ABC, Warszawa.

Gomułowicz A. (2001b), Zasady podatkowe wczoraj i dziś, Dom Wydawniczy ABC, Warszawa.

Gwiazdowski R. (2001), Sprawiedliwość a efektywność opodatkowania. Między progresją a podatkiem liniowym, Centrum im. Adama Smitha, Warszawa.

Hall R.E., Rabushka A. (1998), Podatek liniowy, Dom Wydawniczy ABC, Warszawa.

Homes K.J. (2008), Streamling Personal Income Tax Systems, “Asia-Pacific Bulletin”, IBFD.

Hybka M. (2005), Opodatkowanie dochodów osób fizycznych w Republice Federalnej Niemiec, Wydawnictwo Akademii Ekonomicznej w Poznaniu, Poznań.

Matthews S. (2011), Trends in Top Incomes and their Tax Policy Implications, "OECD Taxation Working Papers", No. 4, OECD Publishing.

Małecka-Ziembińska E. (2012), Efektywność fiskalna podatku dochodowego od osób fizycznych $w$ Polsce, Wydawnictwo Uniwersytetu Ekonomicznego w Poznaniu, Poznań.

Mill J.S. (1966), Zasady ekonomii politycznej i niektóre jej zastosowania do filozofii społecznej, t. 2., Państwowe Wydawnictwo Naukowe, Warszawa.

Moździerz A. (2012), Błędna polityka podatkowa przyczyna wzrastajacych nierówności, [w:] T. Famulska, A. Walasik (red.) Finanse w niestabilnym otoczeniu - dylematy $i$ wyzwania. Finanse Publiczne, Katowice.

OECD (2012), Income Inequality and Growth: The Role of Taxes and Transfers, „OECD Economic Department Policy Notes", no. 9.

OECD (2014a), Fucus on Inequality and Growth. Does Income Inequality Hurt Economic Growth?, OECD Publishing.

OECD (2014b), Income Distribution Database.

OECD (2014c), OECD Tax Database.

OECD (2014d), Taxing Wages. Comparative Tables.

Owens J. (2013), Flat Taxes: Myths and Realities, „Bulletin for International Taxation”, IBFD.

Paturot D., K. Mellbye, B. Brys (2013), Average Pesonal Income Tax Rate and Tax Wedge Progression in OECD Countries, "OECD Taxation Working Papers", no. 15, OECD Publishing.

Smith A. (2007), Badania nad natura i przyczynami bogactwa narodów, t. 2, Wydawnictwo Naukowe PWN, Warszawa.

Stiglitz J. (2004), Ekonomia sektora publicznego, Wydawnictwo Naukowe PWN, Warszawa.

Torres C., Mellbye K., Brys B. (2012), Trends in Personal Income Tax and Employee Social Security Constribution Schedules, "OECD Taxation Working Papers", no. 12, OECD Publishing. 
Matgorzata Mazurek-Chwiejczak

\section{PROGRESJA PODATKU OD DOCHODÓW OSOBISTYCH W PAŃSTWACH OECD W KONTEKŚCIE REDYSTRYBUCJI DOCHODU - KIERUNKI EWOLUCJI}

Streszczenie. Zjawisko nierówności społecznych ma charakter powszechny i trwały. Obecnie w wielu krajach OECD różnice między biegunami bogactwa i biedy osiągnęły najwyższy poziom od 30 lat, zaś odsetek osób zagrożonych ubóstwem sięga nawet 40\%. W takich warunkach konieczne jest prowadzenie szeroko zakrojonej polityki redystrybucyjnej realizowanej za pośrednictwem systemu transferów społecznych, jak i systemu podatkowego. Zasadniczym instrumentem podatkowym wykorzystywanym do wyrównywania poziomu dochodów ludności jest progresywny podatek dochodowy, silniej obciążający osoby lepiej sytuowane. Rolę tę może pełnić również podatek liniowy z kwotą wolną od podatku ustanowioną na odpowiednio wysokim poziomie. W artykule przeprowadzono analizę skali progresji podatków od dochodów osobistych w państwach OECD. W dalszej części dokonano analizy porównawczej podstawowych elementów konstrukcyjnych warunkujących stromość progresji PIT, jakimi są liczba przedziałów skali podatkowej, wysokość stawek obowiązujących w najwyższym przedziale, jak również wysokość dochodu niepodlegająca opodatkowaniu tą daniną.

Słowa kluczowe: podatek dochodowy od osób fizycznych, podatek od dochodów osobistych, progresja, redystrybucja, polityka podatkowa. 